\title{
fMRI evidence supporting the role of memory conflict in the déjà vu experience
}

Josephine A. Urquhart ${ }^{\mathrm{a}}$, Magali H. Sivakumaran ${ }^{\mathrm{a}}$, Jennifer A. Macfarlane ${ }^{\mathrm{b}}$, and Akira R. O’Connor ${ }^{\text {** }}$

${ }^{a}$ School of Psychology \& Neuroscience, University of St Andrews, St Andrews, Scotland UK;

${ }^{b}$ Medical Physics, Ninewells Hospital \& Medical School, Dundee, Scotland UK

*School of Psychology \& Neuroscience, University of St Andrews, St Mary's Quad, South Street, St Andrews, Fife, KY16 9JP, Scotland UK; telephone +44 (0)1334 46 2077; email aro2@st-andrews.ac.uk 


\section{fMRI evidence supporting the role of memory conflict in the déjà vu experience}

Attempts to generate déjà vu experimentally have largely focused on engineering partial familiarity for stimuli, relying on an ensuing, but unprompted evaluation of conflict to generate the experience. Without verification that experimentallygenerated familiarity is accompanied by the awareness of stimulus novelty, these experimental procedures potentially provide an incomplete déjà vu analogue. We used a modified version of the Deese-Roediger-McDermott (DRM) false memory procedure to generate both familiarity and novelty within a déjà vu analogue-we coupled experimentally-generated familiarity with cues indicating that the familiarity was erroneous, using this additional source of mnemonic information to generate cognitive conflict in our participants. We collected fMRI and behavioural data from 21 participants, 16 of whom reported déjà vu. Using univariate contrasts we identified brain regions associated with mnemonic conflict, including the anterior cingulate cortex, medial prefrontal cortex and parietal cortex. This is the first experiment to image an analogue of the déjà vu experience in healthy volunteers. The increased likelihood of déjà vu reports to DRM critical lures correctly identified as "new", and the activation of neural substrates supporting the experience of cognitive conflict during déjà vu, suggest that the resolution of memory conflict may play an integral role in déjà vu.

Keywords: familiarity, conflict, déjà vu, fMRI, medial prefrontal cortex

\section{Introduction}

Déjà vu has been defined as a feeling of recognition combined with an awareness that this feeling is being experienced inappropriately (e.g. Brown, 2004 - "the clash between two simultaneous and opposing mental evaluations: an objective assessment of unfamiliarity juxtaposed with a subjective evaluation of familiarity", p. 2; see also Metcalfe \& Schwartz, 2016, for a discussion of its place within a broader set of spontaneous metacognitive feeling states). It is typically evaluated as peculiar or strange, and this may be because, for a short period of time, a feeling of recognition persists alongside and counter to the awareness that it should not. Retrospective surveys have provided insight into various aspects of the experience: how frequently it occurs; its antecedents; how it is perceived etc. (for reviews see 
Brown, 2003; Brown, 2004; Moulin, 2018). However, due to its typical infrequency, it is likely that weeks or months will separate most respondents' last experiences of déjà vu and their recollections of it, potentially introducing bias and distortion that mischaracterise the experience. Further, surveys are incapable of measuring cognitive and neuroscientific facets of the experience 'as-it-happens', leaving these qualities largely unexplored within the healthy population.

Attempts to generate déjà vu experimentally have largely focused on covertly engineering false or partial familiarity for stimuli, without the conscious awareness of the participant (Brown \& Marsh, 2009; Cleary et al., 2012; Cleary \& Claxton, 2018; Cleary \& Reyes, 2009; Cleary, Ryals, \& Nomi, 2009; O'Connor, Barnier, \& Cox, 2008). For example, Cleary and colleagues innovatively used a virtual reality environment to present participants with configurations of elements within scenes that were either novel or that matched previously visited environments (Cleary et al., 2012). They found that matched environments were rated as both more familiar and more likely to result in déjà vu reports as compared to novel environments. An issue with approaches that solely engineer partial familiarity is that many real-life and experimental situations generate notable feelings of familiarity, but do not result in spontaneous déjà vu reports. For example, Mandler's (1980) 'butcher on the bus' example of contextually inappropriate familiarity, despite its similarity to the empirically engineered situations above, has never previously been likened to déjà vu. This discrepancy points to the possibility that familiarity may be necessary but not sufficient for déjà vu to be experienced. During procedures in which the experimenter engineers familiarity, it is possible that an additional, typically less well-controlled component of the experience - the salience of the familiar item's actual novelty_contributes to the participant's overall experience in such a way as to make the experience of déjà vu much more likely.

Familiarity is clearly an extremely salient, important aspect of the déjà vu experience. We therefore used previous work as a starting point for our own experimental analogue of the experience, engineering a series of situations in which participants would experience both familiarity and an additional awareness of the implausibility of this familiarity (Urquhart \& O'Connor, 2014). Using a modified Deese-Roediger-McDermott (DRM) false memory procedure (Roediger \& McDermott, 1995), we generated familiarity for critical lure words that were semantically linked to previously studied stimuli. Crucially though, this false familiarity was challenged by the presentation of additional mnemonic information that 
participants could use to establish that the words in question were verifiably novel (Fig 1A). Specifically, participants monitored each studied list of words (e.g. wet, snow, winter, ice, freeze, chilly, heat) for whether or not any of them began with a specific letter string $(C O)$. In our critical conditions, none of the studied words began with the string and participants indicated this at the end of studying the study list. Prior to the subsequent test phase, participants were informed of the number of words they had judged as beginning with the letter string (0), and were then asked to make old-new responses regarding a series of targets (e.g. freeze, snow), lures (e.g. speaker, weird) and the critical lure (cold). Importantly even though spreading semantic activation had generated familiarity for the critical lure, participants were able to correctly identify it as novel (a category of stimulus we termed 'resolved critical lures'). Participants reported déjà vu more frequently under these conditions of mnemonic conflict, as compared to critical lures devoid of cues to verifiable novelty (e.g. if the letter string for the above study-test block had been WI; termed 'unresolved critical lures'). This procedure places the unusual experience of memory conflict at the core of the déjà vu experience. Hereafter we refer to its theoretical foundation as the memory conflict account of déjà vu.

Given the relative recency of experimental déjà vu analogue development, no published studies have examined the neural correlates of such experiences in healthy participants. There has however, been extensive investigation of pathological déjà vu in temporal epilepsy, using electrophysiological stimulation and electrocorticographic recording. The general pattern of findings here is that patients with déjà vu as part of their seizure pathology display déjà vu onset triggered by medial temporal lobe (MTL) stimulation (Bartolomei et al., 2012; Vignal, Maillard, McGonigal, \& Chauvel, 2007), demonstrate differences in MTL function during ictal déjà vu experiences relative to controls (Weinand et al., 1994), and show inter-ictal temporal lobe metabolic differences compared to controls and patients for whom déjà vu does not form part of the seizure pathology (Guedj, Aubert, McGonigal, Mundler, \& Bartolomei, 2010). These findings are convergent with those from a structural magnetic resonance imaging (MRI) study of non-epileptic healthy volunteers, which shows reduced MTL grey matter in those who experience déjà vu relative to those who do not (Brázdil et al., 2012). Across these various studies, the literature points to MTL structures established as crucial in signalling the subjective experience of memory retrieval (typically rhinal cortex) as being associated with the occurrence of déjà vu. Indeed, a cognitive study of inter-ictal memory 
performance has demonstrated selective deficits in familiarity but not recollection in patients with déjà vu as part of their seizure pathology (Martin et al., 2012). These cognitive and neuroscientific investigations, whilst speaking to the role of subjective familiarity in déjà vu, tell us little about the importance of novelty and conflict. The significance of the current procedure is that it directly manipulates the salience of stimulus novelty, thereby giving rise to situations in which conflict with familiarity is more or less likely. If familiarity is necessary but not sufficient, and the presence of mnemonic conflict is the key factor determining whether or not déjà vu is experienced, then the neuroscientific markers of déjà vu we observe should be those we recognise as important in conflict monitoring and cognitive control.

In this vein, a positron emission tomography (PET) functional neuroimaging study of epileptics who experience déjà vu favours an account that implicates neural involvement beyond the MTL (Adachi et al., 1999). Glucose metabolism between seizures was measured, with reductions relative to non-déjà vu-experiencing patients observed in the MTL, but also in the parietal cortex, a region implicated in attention to memory (Cabeza et al., 2011; O'Connor, Han, \& Dobbins, 2010). The authors speculate that the parietal cortex could be part of an extended network of regions that integrates the memory experience, driven by the MTL, into the overall perceptual experience. This explanation would be consistent with the memory conflict account of déjà vu, and would be most compellingly illustrated by direct observation of the experience itself. Under such circumstances, stimuli eliciting déjà vu should show activation linked with 'retrieval success' (Konishi, Wheeler, Donaldson, \& Buckner, 2000; McDermott, Gilmore, Nelson, Watson, \& Ojemann, 2017), but also with heightened 'control' reflecting the monitoring demands associated with using metacognitive awareness to countermand prepotent but false familiarity responses (Ridderinkhof, Ullsperger, Crone, \& Nieuwenhuis, 2004).

Here we used the modified DRM procedure to interrogate the importance of memory conflict in the déjà vu experience. To this end, we observed behavioural memory responses, reports of déjà vu occurrence, and fMRI measurement of brain activation. Consistent with the memory conflict account, and in line with previous findings (Urquhart \& O'Connor, 2014), we predicted that resolved critical lures (which can be disambiguated using the additional information as "new") would yield higher déjà vu report rates than other stimuli, including unresolved DRM critical lures (which cannot be disambiguated using the additional 
information and are therefore incorrectly called "old"). We also anticipated that the neural correlates differentiating these déjà vu-triggering stimuli from those less likely to yield déjà $\mathrm{vu}$ responses would be found not in brain regions that support familiarity, but in frontoparietal control regions.

\section{Materials and Methods}

\section{Participants}

Twenty-one right-handed, native English-speaking participants, with normal or corrected-tonormal vision (17 women; age 19-24 years) were included in the main analysis. All participants had previously experienced déjà vu in a naturalistic setting. One participant completed a version of the task that failed to record data from the fourth study-test block, but provided sufficient data from the first three blocks to warrant analysis. One additional participant failed to complete the task due to scanner malfunction. Informed consent was obtained in a manner approved by the University Teaching and Research Ethics Committee at the University of St Andrews (Approval: PS12003), and the Tayside Committee on Medical Research Ethics, Ninewells Hospital and Medical School (Approval: 16/ES/0032).

\section{Stimuli and Procedures}

We generated 32 DRM lists based on a previously published behavioural version of this task (Urquhart \& O’Connor, 2014). Each list comprised 1 critical target (always presented at study; e.g. shade), 14 targets (from which 11 were randomly selected to be presented at study; e.g. door, pane, glass, ledge, sill, house, open, curtain, frame, view, breeze, sash, screen, shutter), 1 critical lure (never presented at study; e.g. window), and 2 unrelated lures (never presented at study; e.g. savior, chapter). The targets and critical lure were all semantically related to one another-the unrelated lures were semantically unrelated to each other and to the semantically related words. So that the associated critical lure for each list could be presented in resolved or unresolved forms, we generated for each list a critical lure letter string (e.g. $W$ ) and a critical target letter string (e.g. $S$ ) — the strings, of length one to three characters, were unique to each word i.e. the letter strings $W$ and $S$ were not used by any of the other lists. Word lists that would contribute resolved critical lures at test, were monitored for the critical lure letter string at study. Thus, a participant would have a high 
likelihood of knowing that the critical lure window had not been presented at study because they had been monitoring the study list for any words beginning with $W$ and responded that no studied words began with that string. Words lists that would contribute unresolved critical lures at test were monitored for the critical target letter string at study. In these lists, the number of times the critical target string $S$ appeared at study would be uninformative in helping the participant decide whether the critical lure window had been presented at study. In this way, we manipulated the mnemonic conflict that would arise in response to highly subjectively familiar critical lures according to whether their recognition status could be resolved using their letter string responses (conflict present) or whether it remained unresolved using this information (conflict absent).

Test lists comprised 8 words: 1 critical target, 2 targets chosen at random from each half of the study list, 1 critical lure, 2 unrelated lures, 2 related lures (target words not shown at study). Word order was pseudorandom, modelled on the procedure proposed by Roediger and McDermott (1995). One of the targets (not the critical target) always occupied test position 1. The critical lure always occupied test position 6-8. Related lures were included alongside critical lures to stop participants completing the recognition task using a solely semantic strategy (i.e. "Unless the meaning relates to doors or windows, the word is new"). The combination of increased familiarity for related lures based on semantic relatedness, and novelty salience-promoting letter strings never corresponding to related lures, meant that these stimuli also acted as a within-list check of whether familiarity alone was enough to generate déjà vu.

Following informed consent and routine safety procedures, participants completed a preexperimental questionnaire, for which they provided demographic information and provided some information about their previous experience of déjà vu, including answering the following question:

Please provide a short account of a 'typical' déjà vu experience you have had. Try to include some detail concerning the following points: emotional intensity of a typical déjà vu experience; duration of a typical déjà vu experience; how a déjà vu experience might typically make you feel about the reliability of your memory.

Participants were then situated in the scanner and underwent structural scans, during which they completed practice versions of the experimental task, and a resting connectivity scan. 
All materials were presented and responses recorded using PCs running MATLAB (The MathWorks Inc., Natick, MA, 2000) and Psychophysics Toolbox (Brainard, 1997). Participants undertook 4 study-test blocks each comprising a $\sim 7.5 \mathrm{~min}$ scanned study phase and a scanned $\sim 6.5 \mathrm{~min}$ test phase. In each study phase, participants were presented with 8 lists: 6 to which the critical lure letter string was allocated, 2 to which the critical target letter string was allocated. For 2 s prior to, and then during each list, a cue indicating the letter string was presented, (e.g. List $1-$ ' $w$ '). Each list word was presented onscreen for 3.5s with a $0.5 \mathrm{~s}$ inter-stimulus-interval (ITI). Following the presentation of all 12 words in each study list, participants were prompted for a response (e.g. Number of ' $w$ 's?) and indicated it using their left hand and a four-button response box with buttons corresponding to 1,2, 3 and 4+. The response window was $6 \mathrm{~s}$. Overall accuracy was $95.2 \%(\mathrm{SE}=1.4 \%)$.

In each test phase, participants were presented with 8 lists in the same order as at study. For $2 \mathrm{~s}$ prior to, and then during each list, a cue presented the previously monitored letter string and participant response (e.g. List $1-$ ' $w$ ' reported 0 times). Each list word was onscreen for 5.5s during which time participants reported their recognition confidence using their left hand and a four-button response box-new-H[igh], new-L[ow], old-L, old-H. We implemented a number of procedures recommended for the minimisation of demand characteristics during the assessment of subjective memory experiences, including the use of an optional response to indicate the occurrence of the sensation, and the provision of an operational definition of déjà vu prior to each test block (Jersakova, Moulin, \& O’Connor, 2016; O'Connor \& Moulin, 2010). Thus, rather than being prompted for a déjà vu response for each stimulus, participants optionally indicated whether or not they experienced déjà vu using a right-hand response box response, switching the default déjà vu status from deja vu: no to deja vu: yes (and back if they responded in error). A definition of déjà vu as follows, was provided in the experiment instructions and in the intervening period between study and test phases: Déjà vu is defined as a feeling of familiarity coupled with the awareness that this familiarity is incorrect. Please consider this definition and your own personal experience of déjà vu when reporting the experience. There was an ITI of 0.5s. For each participant, the 32 lists were randomised across the 4 study-test blocks, with the 6 critical lure strings and 2 critical target strings also randomly allocated to lists within each block. Total time in the scanner was approximately one hour. 
Finally, participants completed a post-experimental questionnaire, in which they answered the following question:

Please provide a short account of your déjà vu experience(s) during the experiment. Try to include some detail concerning the following points: emotional intensity of déjà vu experience(s); duration of déjà vu experience(s); how the déjà vu experience (s) made you feel about the reliability of your memory.

\section{fMRI Acquisition, Preprocessing and Analysis}

Scanning was performed on a 3T Siemens Prisma Fit whole-body MRI scanner (Siemens Medical Solutions, Erlangen, Germany) using a 20 channel head and neck coil. Whole brain functional data were acquired using a descending echo-planar pulse sequence (TR $=2000 \mathrm{~ms}$, $\mathrm{TE}=30 \mathrm{~ms}$, 90 degree flip angle, 36 axial slices parallel to the AC-PC plane with $3.5 \mathrm{~mm}$ isotropic voxels, $0.5 \mathrm{~mm}$ inter-slice gap). Head motion was minimised using foam padding. All BOLD data were processed with SPM8 (Wellcome Department of Imaging Neuroscience, London). Slice acquisition timing correction was carried out by temporally resampling relative to the middle slice collected, followed by rigid body motion correction. Functional volumes were then spatially normalised to a canonical echo-planar template using 12parameter affine and cosine basis transformations, and resampled to $3 \mathrm{~mm}$ isotropic voxels. Volumes were then spatially smoothed with a $6 \mathrm{~mm}$ Gaussian kernel.

Participants were treated as a random effect and volumes as a temporally correlated time series. Summary amplitudes were modelled in SPM8 by convolving a canonical hemodynamic response function with a series of delta functions marking the onset of each condition of interest. The $\beta$ estimates of the best-fitting canonical hemodynamic response function for each condition were used in pairwise contrasts and stored as a separate image for each participant. These contrast images were tested against the null hypothesis of no difference between contrast conditions using one-tailed, repeated measures t-tests. All contrasts on the full sample were tested at a one-sided $p$-threshold of .0001 using a statistically-derived cluster extent to yield a family-wise error alpha of .05. Cluster extent thresholds were determined using AFNI's 3dClustSim (Cox, 1996; Cox \& Hyde, 1997; Gold et al., 1998), and entered into SPM8's output interface to yield the results reported here. $p$ thresholds and cluster extent thresholds are noted in figure legends and table notes. In this paper, we have disregarded response confidence (high and low confidence correct responses 
were modelled together), meaning that all effects reported here are unbiased with regard to response confidence.

Unthresholded $t$-maps for all univariate contrasts described in the Results have been uploaded to NeuroVault (Gorgolewski et al., 2015) and are available under CC0 license at:

https://neurovault.org/collections/4094/. 


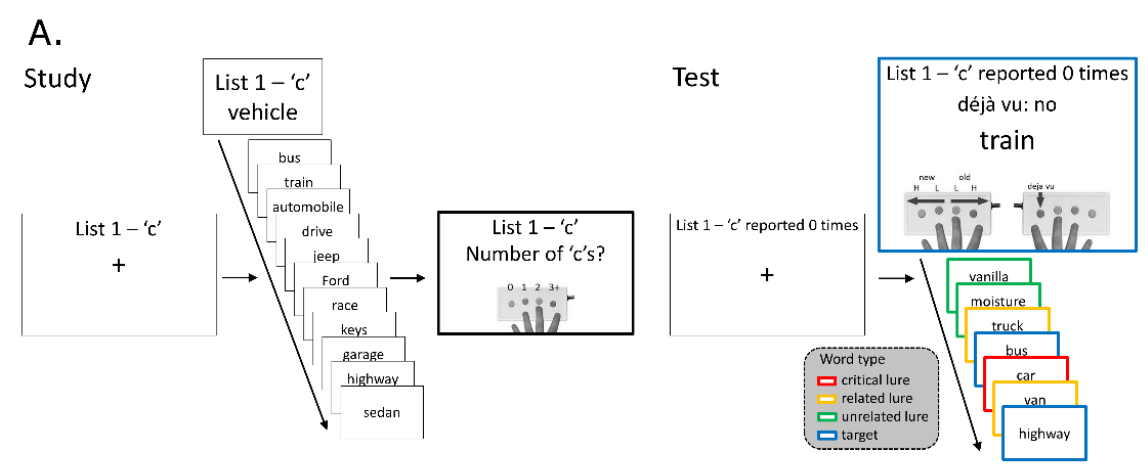

B.

C.
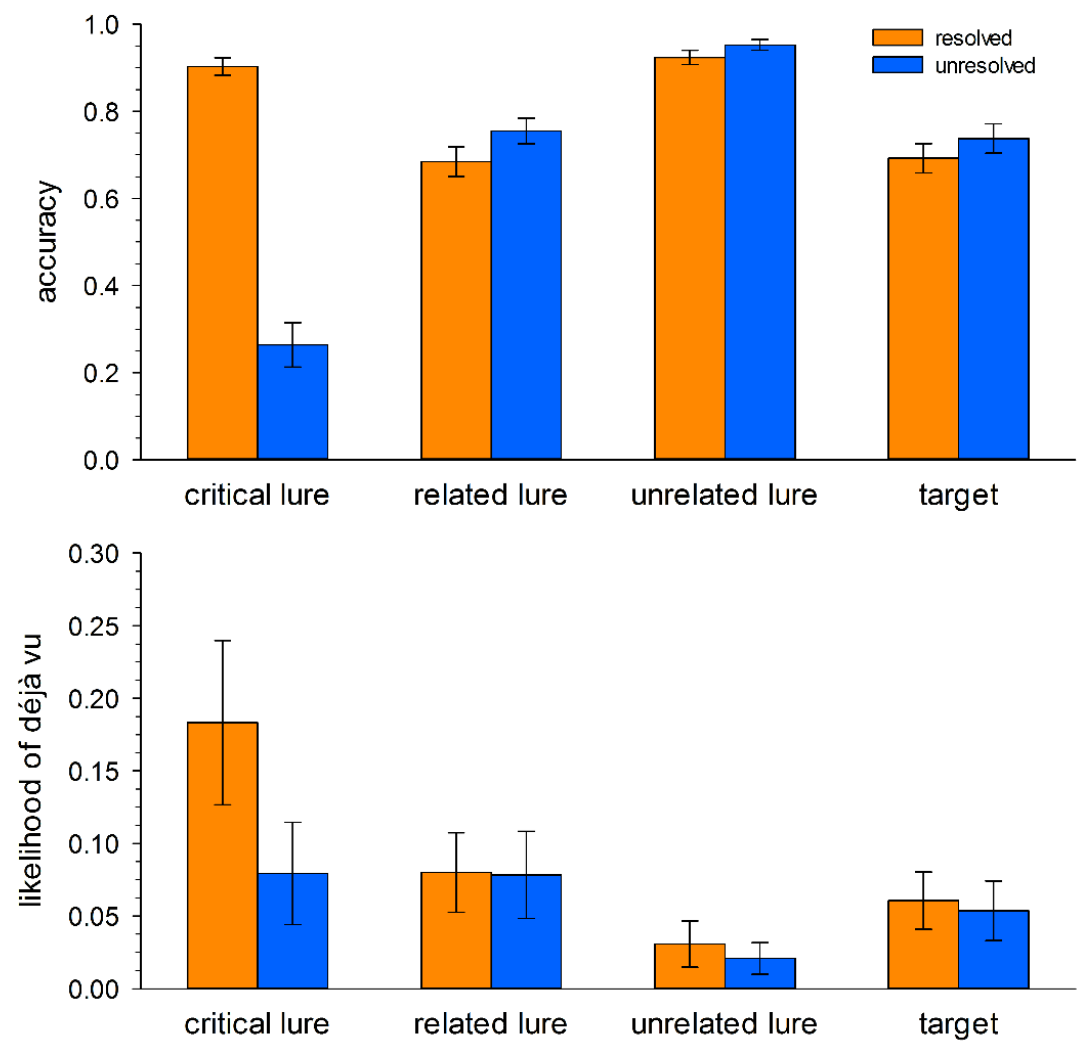

Figure 1: Study Design Schematic, Memory Accuracy and Likelihood of Déjà vu. Panel A shows an example resolved study-test list within the modified DRM procedure. In the study list, the participant is instructed to monitor study words for whether or not they begin with a letter string: C. After the study list has been presented, they respond with the number of words beginning with that letter string: " 0 ". This response would be non-zero in an unresolved list i.e. at least one target would begin with the letter string. At test they are continually presented with the string and their previous response, and are presented with various stimuli (the word types were not apparent to participants but are presented in the schematic), including a critical lure, which begins with the to-be-monitored letter string. The critical lure appears subjectively familiar but is known from the information provided in response to the critical lure letter string to be novel — a situation in which déjà vu is anticipated. Participants can indicate déjà vu status for each stimulus using the right-hand button box, and render a memory response using the left-hand button box. Panels B and C respectively show response accuracy déjà vu likelihood and according to word and list type. Error bars are standard errors of the mean. 


\section{Results}

Overall recognition accuracy at test was $.765(\mathrm{SE}=.012)$. However accuracy was higher for resolved than unresolved critical lures (Fig 1B), $t(20)=11.12, p<.001, d=2.59$, validating the utility of both the DRM critical lures in generating compelling false familiarity (unresolved critical lures), and the additional mnemonic information in aiding memory conflict resolution (resolved critical lures). It is worth noting here that accuracy for unresolved critical lures deviated from the accuracy for all other stimulus classes, including related lures, whilst resolved critical lures were responded to as accurately as these other stimulus classes. In fact, resolved critical lures were responded to more accurately than related lures from either list type, $\mathrm{F}(2,40)=22.22, p<.001, \eta_{p}{ }^{2}=.526$ with $p<.001$ for both pairwise comparisons involving resolved critical lures - the provision of novelty cues left participants in no doubt as to the novelty of even these highly semantically related stimuli. These behavioural data provide a strong foundation to determine whether false familiarity alone is sufficient to generate déjà vu, which would be evidenced by both resolved and unresolved critical lures showing higher déjà vu rates than other stimuli. Alternatively, if mnemonic conflict resolution is an important component of the déjà vu experience, then higher déjà vu rates would be observed in resolved critical lures alone.

Déjà vu was reported at least once by 16 participants (76\% of the sample). Reports were most numerous for resolved critical lures (Fig 1C; though the difference from unresolved critical lures was nonsignificant, $\mathrm{t}[20]=1.51, p=.147, d=0.28$ ), replicating previous behavioural findings (Urquhart \& O’Connor, 2014). Overall there were different patterns of responding across the two varieties of critical lure. Resolved critical lures were highly likely to be correctly identified as "new" yet yielded numerically higher déjà vu report rates than all other categories of stimulus, whilst unresolved critical lures were highly likely to be incorrectly identified as "old", but yielded lower déjà vu report rates than resolved critical lures.

We followed up these behavioural analyses by examining the fMRI data. We first demonstrate that resolved critical lures show an associated pattern of neural activation consistent with the subjective experience of familiarity, despite behavioural responding indicating otherwise. We then localise the neural substrates of conflict resolution and, in a restricted sub-sample, the neural substrates supporting the déjà vu experience. 
To illustrate the pattern of brain activation associated with retrieval, and contributed to by familiarity, we first examined the 'retrieval success' contrast (targets vs lures [correct responses only; excluding resolved and unresolved critical lures]; Figure 2A; Table 1; the reverse of this contrast revealed no suprathreshold activations). Standard features of this contrast, including lateral parietal and lateral prefrontal cortex (PFC) activation were apparent within a large swathe of activations. We next examined a 'false familiarity' contrast (resolved critical lures vs lures [correct responses only; excluding resolved and unresolved critical lures]; Figure 2B; Table 2; the reverse of this contrast revealed one 17 voxel suprathreshold cluster in orbitofrontal cortex with cluster maximum [-36, 32, -17]). While it has been established that standard DRM critical lures display a neural signature similar to targets correctly identified as “old” (McDermott et al., 2017), those items are experienced and responded to as old. Here, we aimed to establish whether our resolved critical lures-which elicit "new" responses - would also show this pattern characteristic of subjective familiarity integral to both theoretical accounts being adjudicated. Despite their "new" responses, resolved critical lures also show the hallmarks of retrieval, characterised by parietal cortex and lateral PFC activation. While the extent of retrieval success activation was generally larger, the resolved critical lure activation was more extensive within bilateral parietal surfaces and along the frontal midline (Figure 2C). These differences in the extent of activations suggest that the putative mnemonic conflict present in resolved critical lures may be supported by a set of frontoparietal regions not recruited during standard retrieval success. 

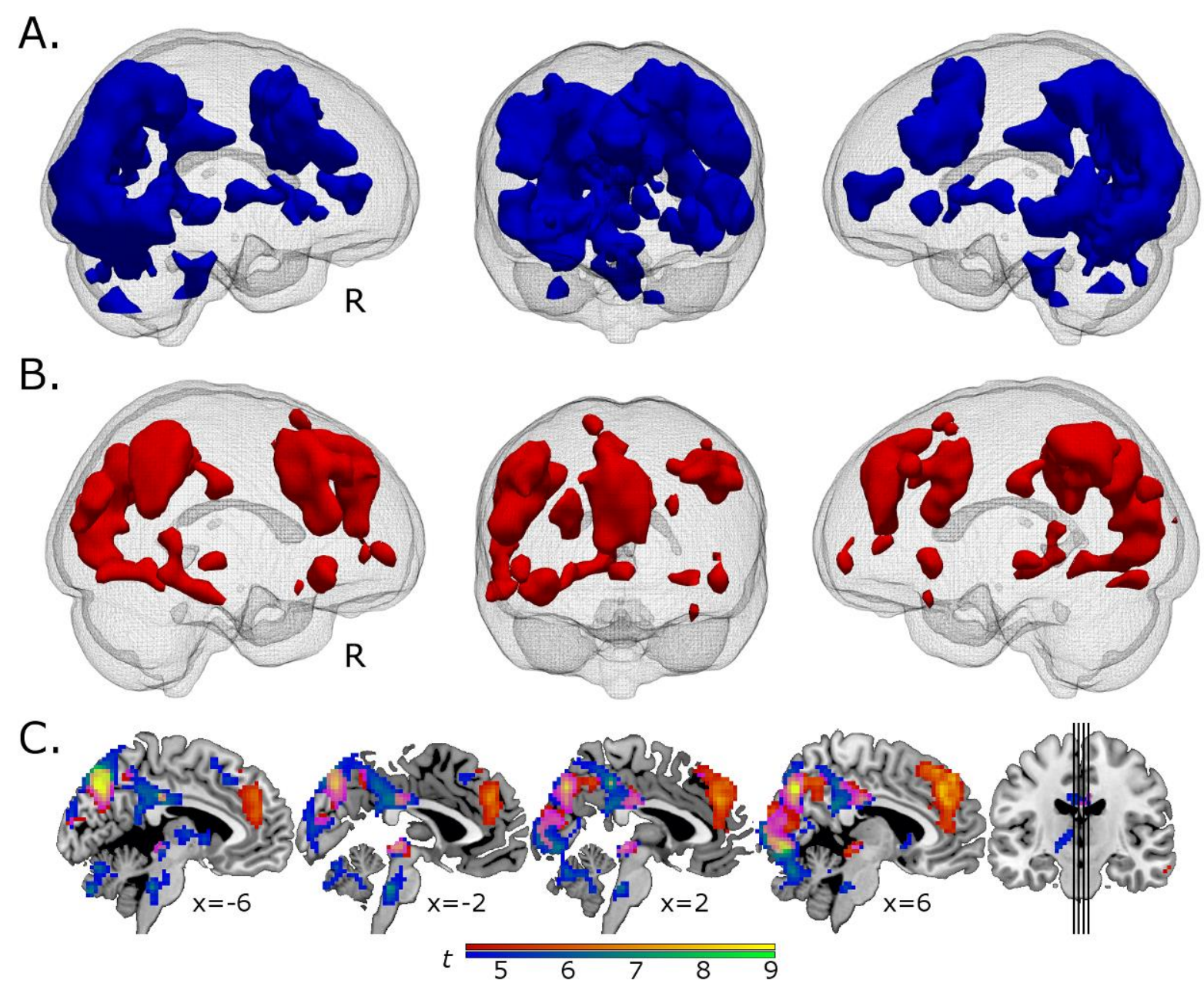

Figure 2. 'Retrieval Success' (Targets vs Lures) and 'False Familiarity' (Critical Lures vs Lures) Contrasts. Panel A shows a smoothed rendering of highest suprathreshold cluster density (blue) for targets vs lures (correct responses only; excluding critical lures). The $t$ threshold contributing to this rendering was set at .0001 , and the cluster threshold determined to be 16. Panel B shows a smoothed rendering of highest suprathreshold cluster density (red) for resolved critical lures (critical lures for which disambiguating information indicative of the words' novelty led to correct "new" responding) vs lures (correct responses only; excluding critical lures). The $t$-threshold here was set at .0001 , and the cluster threshold determined to be 17 . Panel $\mathrm{C}$ shows the convergence of $t$-maps corresponding to the above contrasts plotted on sagittal sections along the midline (x co-ordinates below each section). Targets vs lures is shown in cool colours, resolved critical lures vs lures in warm colours, overlap in purple/yellow. Glass brains shown in Panels A and B, and Figure 3 rendered using ParaView (Ahrens, Geveci, Law, Hansen, \& Johnson, 2005) and ITK-SNAP (Yushkevich et al., 2006) following procedures based on (Madan, 2015). 
Table 1: Activations for Targets vs Lures (Correct Responses Only; Excluding Critical Lures)

\begin{tabular}{|c|c|c|c|c|c|c|c|}
\hline Region & Lat. & BA & $x$ & $y$ & $z$ & Vox. & $Z$ score \\
\hline \multicolumn{8}{|l|}{$\begin{array}{l}\text { Cerebellum, Occipital, Parietal, } \\
\text { Temporal Cortex }\end{array}$} \\
\hline $\begin{array}{l}\text { calcarine sulcus, } \\
\text { anterior/posterior cerebellum, } \\
\text { cuneus, fusiform, IPC, ITG, } \\
\text { lingual gyrus, MTG, PCC, } \\
\text { precuneus, SPC, STG }\end{array}$ & $\mathrm{L} / \mathrm{R}$ & $\begin{array}{l}2,7,17 \\
18,19,20 \\
21,22,23 \\
26,37,39 \\
40,41\end{array}$ & 24 & -73 & -8 & 7575 & 6.16 \\
\hline \multicolumn{8}{|l|}{ Frontal Cortex } \\
\hline $\begin{array}{l}\text { IFG, MFG, precentral gyrus, } \\
\text { SMA, mPFC }\end{array}$ & $\mathrm{L}$ & $\begin{array}{l}4,6,8,32 \\
44,45,48\end{array}$ & -45 & 5 & 52 & 998 & 5.62 \\
\hline IFG, precentral gyrus, mPFC & $\mathrm{R}$ & $\begin{array}{l}6,8,9,32 \\
44,45,48\end{array}$ & 42 & 23 & 25 & 591 & 5.34 \\
\hline insula & $\mathrm{L}$ & 47 & -30 & 20 & -2 & 63 & 4.76 \\
\hline IFG, OFC, aPFC & $\mathrm{L}$ & $10,45,46$ & -45 & 50 & -8 & 176 & 4.64 \\
\hline OFC & $\mathrm{R}$ & 47 & 33 & 26 & -8 & 24 & 4.17 \\
\hline $\begin{array}{l}\text { Brainstem, Central, Cerebellum } \\
\text { anterior cerebellum, } \\
\text { midbrain, thalamus }\end{array}$ & $\mathrm{L} / \mathrm{R}$ & - & -3 & -34 & -35 & 170 & 5.06 \\
\hline \multicolumn{8}{|l|}{ Brainstem } \\
\hline \multicolumn{8}{|l|}{ Central } \\
\hline caudate & $\mathrm{R}$ & - & 12 & 11 & -5 & 59 & 4.30 \\
\hline
\end{tabular}

Note: Listed regions are SPM clusters containing at least 16 significant voxels one-sided $p$ threshold of .0001 using a statistically-derived cluster extent to yield a family-wise error alpha of .05. $x, y$, and $z$ coordinates refer to cluster maxima in MNI space. Lat., Laterality; BA, approximate Brodmann's area; Vox., number of significant voxels; ACC, anterior cingulate cortex; IPC, inferior parietal cortex; ITG, inferior temporal gyrus; MTG, middle temporal gyrus; OFC, orbitofrontal cortex; PCC, posterior cingulate cortex; aPFC, anterior prefrontal cortex; mPFC, medial prefrontal cortex; SMA, supplementary motor area; SPC, superior parietal cortex; STG, superior temporal gyrus. Coordinates are in MNI space. 
Table 2: Activations for Resolved Critical Lures vs Lures (Correct Responses Only)

\begin{tabular}{|c|c|c|c|c|c|c|c|}
\hline Region & Lat. & $\mathbf{B A}$ & $\mathbf{x}$ & $\mathbf{y}$ & $\mathbf{Z}$ & Vox. & Z score \\
\hline \multicolumn{8}{|l|}{ Frontal Cortex } \\
\hline $\begin{array}{l}\text { ACC, SMA, MFG, mPFC, } \\
\text { SFG }\end{array}$ & $\mathrm{L} / \mathrm{R}$ & $\begin{array}{l}6,8,9,10 \\
32\end{array}$ & 9 & 41 & 37 & 628 & 5.69 \\
\hline precentral gyrus, IFG, MFG & $\mathrm{R}$ & $\begin{array}{l}6,9,44 \\
46,48\end{array}$ & 51 & 23 & 40 & 485 & 5.22 \\
\hline insula & $\mathrm{R}$ & 47 & 36 & 20 & -8 & 92 & 5.17 \\
\hline MFG, OFC & $\mathrm{L}$ & 46,47 & -39 & 53 & 10 & 37 & 4.71 \\
\hline MFG & $\mathrm{L}$ & 44 & -45 & 26 & 37 & 53 & 4.49 \\
\hline $\mathrm{OFC}$ & $\mathrm{L}$ & 38 & -30 & 17 & -20 & 22 & 4.46 \\
\hline MFG & $\mathrm{R}$ & 46 & 42 & 47 & 1 & 37 & 4.35 \\
\hline MFG & $\mathrm{L}$ & 6 & -39 & 8 & 55 & 17 & 4.31 \\
\hline \multicolumn{8}{|l|}{ Parietal Cortex } \\
\hline IPC & $\mathrm{R}$ & $22,39,40$ & -5 & -55 & 40 & 606 & 5.15 \\
\hline IPC, SPC & $\mathrm{L}$ & 40,7 & -57 & -52 & 43 & 412 & 4.99 \\
\hline \multicolumn{8}{|l|}{ Occipital Cortex } \\
\hline $\begin{array}{l}\text { calcarine sulcus, cuneus, } \\
\text { fusiform, lingual gyrus, } \\
\text { precuneus }\end{array}$ & $\mathrm{L} / \mathrm{R}$ & $\begin{array}{l}7,17,18 \\
19,37,39\end{array}$ & -12 & -70 & 31 & 1167 & 5.12 \\
\hline occipital cortex & $\mathrm{L}$ & 39 & -27 & -79 & 28 & 40 & 4.44 \\
\hline PCC & $\mathrm{L} / \mathrm{R}$ & 23 & 3 & -22 & 34 & 94 & 4.38 \\
\hline fusiform, lingual gyrus & $\mathrm{L}$ & 19,37 & -27 & -76 & -11 & 75 & 4.33 \\
\hline \multicolumn{8}{|l|}{ Brainstem } \\
\hline midbrain & $\mathrm{L} / \mathrm{R}$ & - & 0 & -31 & -5 & 61 & 5.04 \\
\hline $\begin{array}{r}\text { Temporal Cortex } \\
\text { ITG, MTG }\end{array}$ & & 2 210 & 60 & 31 & 17 & 162 & 4.89 \\
\hline
\end{tabular}

Note: Listed regions are SPM clusters containing at least 17 significant voxels one-sided $p$ threshold of $.0001 \mathrm{using}$ a statistically-derived cluster extent to yield a family-wise error alpha of .05. $x, y$, and $z$ coordinates refer to cluster maxima in MNI space. Lat., Laterality; BA, approximate Brodmann's area; Vox., number of significant voxels; ACC, anterior cingulate cortex; IFG, inferior frontal gyrus; IPC, inferior parietal cortex; ITG, inferior temporal gyrus; MFG, middle frontal gyrus; MTG, middle temporal gyrus; OFC, orbitofrontal cortex; PCC, posterior cingulate cortex; aPFC, anterior prefrontal cortex; mPFC, medial prefrontal cortex; SFG, superior frontal gyrus; SPC, superior parietal cortex. 
To isolate and further explore the substrates differentially supporting retrieval and conflict resolution, we used a whole-brain 'conflict resolution' contrast: resolved critical lures vs targets (Figure 3A; Table 3; the reverse of this contrast yielded 3 suprathreshold clusters, 23 voxels in orbitofrontal cortex with cluster maximum [-33, 32, -17], 27 voxels in precuneus with cluster maximum $[-3,-52,10]$ and 30 voxels in calcarine sulcus with cluster maximum $[18,-91,1])$. At this stage it is worth noting that another contrast could potentially have yielded better controlled brain maps: resolved critical lures vs unresolved critical lures. Unfortunately we designed the experiment so that lists containing unresolved critical lures would act primarily as 'catch' lists, reducing the likelihood of participants spotting patterns in the DRM list presentation. We therefore had too few trials including unresolved critical lures to analyse in a statistical parametric map (SPM). Therefore, our rationale for contrasting resolved critical lures against targets was that both sides of the contrast yield subjective oldness (evidenced in Figure 1A and 1B) leaving the successful conflict resolution necessary to render a "new" response present in only the resolved critical lures. This set of activations falls within the frontoparietal network mentioned above (including lateral parietal and medial prefrontal regions), a network known to support the synthesis of incongruent mnemonic information into an eventual memory decision (Jaeger, Konkel, \& Dobbins, 2013; Mill, Cavin, \& O'Connor, 2015; O'Connor, Han, et al., 2010).

Finally, an ideal contrast in which to examine neural correlates of experimentally-generated déjà vu was resolved critical lures which had elicited déjà vu reports vs. those which had not. Unfortunately there were only six participants in which at least two of the four scans contributed to this contrast, making it difficult to generalise from, even to the current sample. For illustrative purposes, we show the contrast thresholded at $p<.01,100$ contiguous voxels (Figure 3B; Table 4; the reverse of this contrast yielded no suprathreshold clusters). Cortical activations differentiating the contrast conditions at this threshold fell mainly along the midline, supplemented by occipital cortex. Posterior aspects of the midline activation survived thresholding at a threshold of $p<.001,5$ contiguous voxels. 

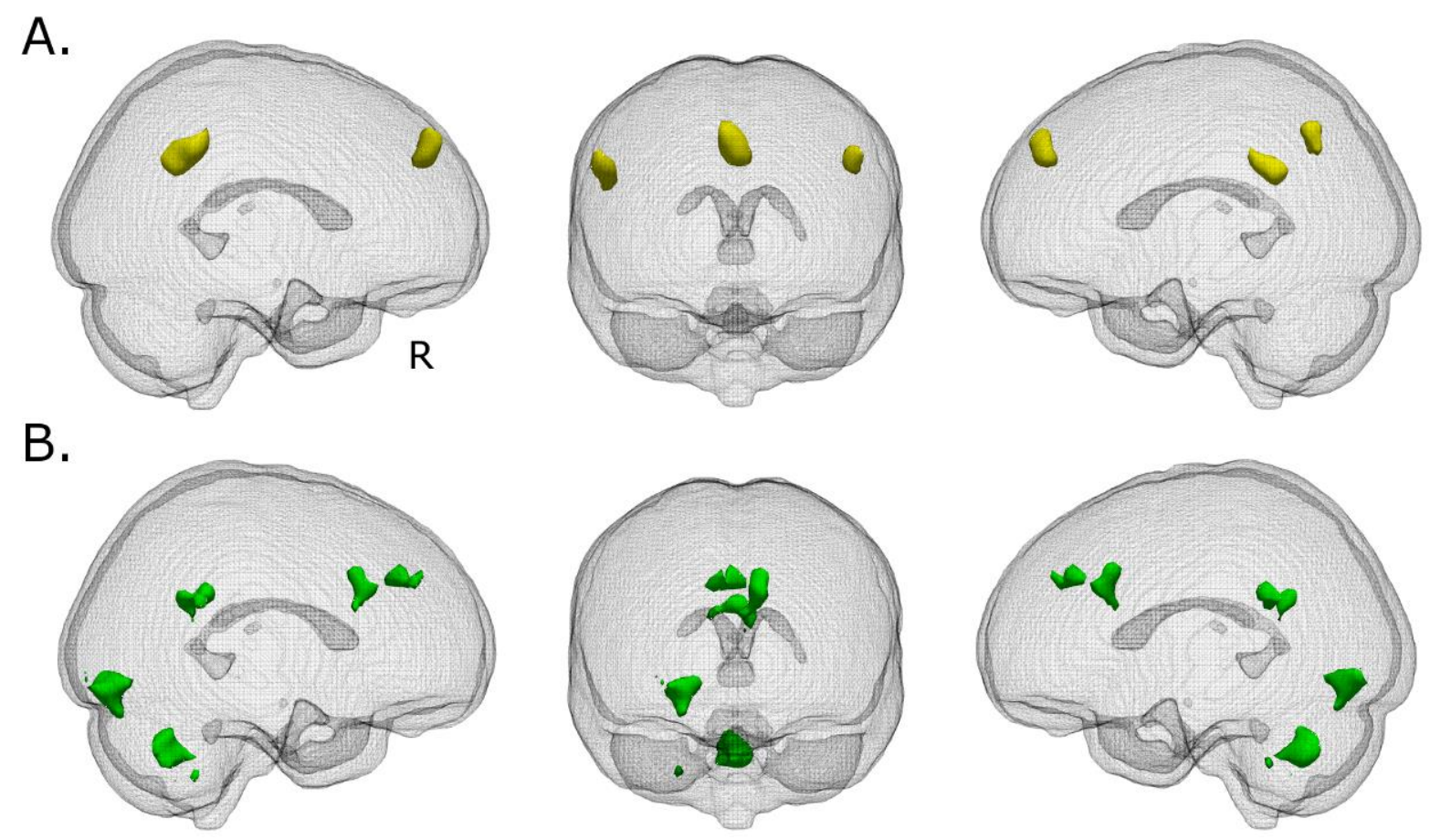

Figure 3. 'Conflict Resolution' (Resolved Critical Lures vs Targets) and 'Déjà vu' (Déjà vu vs No Déjà vu in Resolved Critical Lures) Contrasts. Panel A shows a smoothed rendering of highest suprathreshold cluster density (yellow) for resolved critical lures vs targets. The $t$-threshold here was set at .0001, and the cluster threshold determined to be 14 . Panel B shows a smoothed rendering of highest suprathreshold cluster density (green) for resolved critical lures eliciting déjà vu reports vs those that did not in a subsample of six participants in whom the contrast was possible in at least two of the four experimental scans. Given the small subsample used here, we used an arbitrary (not determined using the procedures described in the Methods) $t$-threshold of .01 and cluster extent threshold of 100 . 
Table 3: Activations for Resolved Critical Lures vs Targets (Correct Responses Only)

\begin{tabular}{|c|c|c|c|c|c|c|c|}
\hline Region & Lat. & $\mathbf{B A}$ & $x$ & $\bar{y}$ & $z$ & Vox. & $Z$ score \\
\hline \multicolumn{8}{|l|}{ Parietal Cortex } \\
\hline IPC & $\mathrm{R}$ & 40,22 & 60 & -46 & 36 & 77 & 4.72 \\
\hline IPC & $\mathrm{L}$ & 39 & -54 & -61 & 3 & 44 & 4.33 \\
\hline Frontal Cortex & & & & & & & \\
\hline $\mathrm{mPFC}$ & $\mathrm{L} / \mathrm{R}$ & 9 & 6 & 53 & 40 & 61 & 4.59 \\
\hline
\end{tabular}

Note: Listed regions are SPM clusters containing at least 14 significant voxels one-sided $p$ threshold of .0001 using a statistically-derived cluster extent to yield a family-wise error alpha of .05. $x, y$, and $z$ coordinates refer to cluster maxima in MNI space. Lat., Laterality; BA, approximate Brodmann's area; Vox., number of significant voxels; IPC, inferior parietal cortex; mPFC, medial prefrontal cortex.

Table 4: Activations for Déjà vu vs No Déjà vu in Resolved Critical Lures

\begin{tabular}{llllllll}
\hline Region & Lat. & BA & $\boldsymbol{x}$ & $\boldsymbol{y}$ & $\boldsymbol{z}$ & Vox. & $\boldsymbol{Z}$ score \\
\hline $\begin{array}{l}\text { Cerebellum } \\
\quad \text { posterior cerebellum }\end{array}$ & L/R & - & 0 & -67 & -38 & 145 & 4.40 \\
$\begin{array}{c}\text { Cerebellum, Occipital Cortex } \\
\quad \text { anterior cerebellum, fusiform }\end{array}$ & $\mathrm{R}$ & 18,19 & 27 & -82 & -14 & 108 & 3.85 \\
$\begin{array}{l}\text { Frontal Cortex } \\
\quad \text { ACC, mPFC }\end{array}$ & L/R & $9,24,32$ & 3 & 32 & 37 & 109 & 3.84 \\
$\begin{array}{l}\text { Parietal Cortex } \\
\quad \text { PCC, precuneus }\end{array}$ & L/R & $23,26,29$ & -3 & -46 & 28 & 100 & 3.56 \\
\hline
\end{tabular}

Note: Listed regions are SPM clusters containing at least 100 significant voxels at the threshold $p<.01 . x, y$, and $z$ coordinates refer to cluster maxima in MNI space. Lat., Laterality; BA, approximate Brodmann's area; Vox., number of significant voxels; ACC, anterior cingulate cortex; PCC, posterior cingulate cortex; mPFC, medial prefrontal cortex. 


\section{Discussion}

This is the first procedure to functionally image an experimental analogue of déjà vu. We used a modified DRM procedure to engineer both subjective familiarity, and the awareness that this familiarity was incorrect, in resolved critical lures. Mnemonic conflict caused by this procedure activated brain regions also supporting the differentiation of resolved critical lures from targets. Our findings are consistent with a memory conflict account of déjà vu, which requires familiarity, and crucially, an awareness of the inappropriateness of the familiarity, to be simultaneously experienced in order for déjà vu to arise.

A critical component of the déjà vu analogue used here, refining analogues used in recent years (Brown \& Marsh, 2009; Cleary et al., 2012; Cleary \& Reyes, 2009; Cleary et al., 2009; O'Connor et al., 2008), is the mnemonic conflict generated by the presentation of information by which the novelty of critical lures can be verified. Conflict was essential in eliciting activation in brain regions with an established role in cognitive control such as medial aspects of the prefrontal cortex, including anterior cingulate (Botvinick, Nystrom, Fissell, Carter, \& Cohen, 1999; Carter \& van Veen, 2007; Metcalfe \& Schwartz, 2016; Ridderinkhof et al., 2004), and both medial and lateral parietal cortex (Bunge, Hazeltine, Scanlon, Rosen, \& Gabrieli, 2002; O'Connor, Han, et al., 2010; Schumacher, Elston, \& D'Esposito, 2003). As to whether these memory conflict-linked regions are the neural substrates underpinning the déjà vu experience (or even whether such a modular substrate exists), this remains to be elucidated. It is certainly the case that electrophysiological studies eliciting déjà vu-like experiences in patients have had greatest success via stimulation of the medial temporal, rather than prefrontal, cortex (Bartolomei et al., 2012; Vignal et al., 2007), though this does not rule out the role of medial prefrontal regions integrating mnemonic information from various sources including the medial temporal cortex, and signalling a mismatch experienced phenomenologically as déjà vu (Metcalfe \& Schwartz, 2016; O'Connor, Lever, \& Moulin, 2010).

An impediment to stronger conclusions on the role of the neural substrates of mnemonic conflict in déjà vu formation results from the constraints upon the imaging analyses we were able to conduct. As noted in the Results section, our univariate contrast identifying the neural correlates of conflict resolution (Figure 3A) are not those we would have chosen had trial counts been favourable. Similarly, our univariate contrast identifying the neural correlates of 
déjà vu (Figure 3B) was performed at a lax statistical threshold due to the fact that we were only able to identify six participants whose data could contribute to the contrast. As such, we present neuroimaging data that corroborate the behavioural findings, but the interpretation of which relies on reverse inference from sub-optimal contrasts. The perils of reverse inference from neuroimaging data have been well documented (e.g. Poldrack, 2011). Nonetheless, we argue that the neuroimaging and behavioural data together provide convergent evidence pointing towards the potential importance of mnemonic conflict resolution in déjà vu. It remains to be seen whether the current analogue can be sufficiently modified to improve its suitability for neuroimaging analyses, or whether alternative procedures drawing on the principles outlined here will have to be used, but we believe that the current procedure is an important first step in understanding the cognitive and neural basis of the déjà vu experience.

The precise nature of cognitive conflict within the déjà vu experience is also an issue that requires more investigation. Metcalfe and Schwartz (2016) make a compelling argument that 'spontaneous metacognitive feeling states' such as déjà vu arise when the current subjective experience is mismatched with the cognitive knowledge state (i.e. what one knows to be true about the subject of the experience). Given that our neuroscientific data sit alongside behavioural results which indicate that déjà vu is more likely to be reported for correctly resolved critical lures (falsely familiar new items that are nonetheless responded to as "new"), we suggest that déjà vu is associated not only with the presence of mnemonic conflict, but its resolution in favour of the evaluation that the associated stimulus is new. Placing conflict resolution as central to this experimental analogue draws it in line with naturalistic déjà vu experiences which tend to be resolved in favour of a novelty evaluation (i.e. they do not result in false recognition and subsequent behaviour change, in contrast with clinical manifestations of déjà vécu e.g. Moulin, Conway, Thompson, James, \& Jones, 2005). Nevertheless, the most powerful test of this proposal would be to directly contrast stimuli that elicit mnemonic conflict according to whether they are resolved in favour of familiarity or novelty. Given the relatively infrequent occurrence of conflict stimuli within this procedure (only once per test list), it is unlikely that this analogue could be extended to yield enough trials-of-interest for this contrast. It is therefore necessary to continue developing and refining experimental analogues of déjà vu if we are to answer this critical question.

The development and refinement of déjà vu analogues is an ongoing endeavour that various research groups have made good progress on over recent years. Given the successes that have 
been reported in the literature, our proposed procedural refinement raises the question of how previous déjà vu analogues that have not explicitly presented a novelty cue have succeeded in generating déjà vu (e.g. Brown \& Marsh, 2009; Cleary et al., 2012; Cleary \& Claxton, 2018; Cleary \& Reyes, 2009; Cleary et al., 2009; O'Connor et al., 2008). Amongst other factors, we suggest that the absence of a novelty cue does not prevent the participant from arriving at their own feeling of novelty e.g. by noticing distinctive aspects of the stimulus they know they would have recollected. The advance within the current approach, therefore, is not that it redefines experimentally-generated déjà vu as one resulting from a resolved conflict between familiarity and novelty, but that it makes salient both of these potential decisions (rather than just one) leaving the participant with less to do to arrive at a situation in which conflict must be resolved. In line with this conclusion, we previously manipulated the novelty cue presented during this procedure, finding that novelty cue removal reduced déjà vu reporting (Urquhart \& O'Connor, 2014). As a further test of this idea, it would presumably be straightforward to incorporate an explicit novelty cue into the partial familiarity procedures cited above, to observe whether or not this makes a difference to déjà vu reporting rates.

A major challenge for research on experimental analogues of déjà vu lies in establishing the veracity of the experimental procedures in generating the experience, rather than simply providing a plausible context that enables demand characteristic-driven reports to be made more easily. Given the difficulty eliminating demand characteristic-driven déjà vu reports, even when experimenters administer procedures that do not generate the experience (Jersakova et al., 2016), it is important to collect and examine evidence that both supports and questions the qualities of the generated experience in relation to real-world experiences participants will previously have had. To this end, we recruited only participants who had previous experience of déjà vu outside the experimental setting, and asked participants to first elaborate on naturalistic déjà vu experiences, and then on the experimentally-generated ones in comparison.

The results of informal analyses of the descriptions of experimentally-generated déjà vu were similar to those of systematic analyses detailed previously (Urquhart \& O'Connor, 2014), namely that participants noted the specificity of the experimental déjà vu experience to the experimental situation (e.g. "... when that word was in the test phase it was familiar in an expected way, rather than the surreal experience of déjà vu in everyday like, when the experience is sudden and eerie."), generally felt the experience to be shorter in duration (e.g. 
"This déjà vu lasted less time than in previous situations..."), and found the intensity to be different compared to the everyday experience. There was variation in reports of intensity, with most participants reporting it to be less intense, though two participants reported an elevated level of intensity (e.g. "it was possibly more intense because I had a strong awareness of all of my body and wasn't as relaxed as I normally am.”). Overall, participants could point to differences with naturalistic déjà vu, but did not identify differences that warrant reconsideration of the experimental results. More positively, if déjà vu is a frontally mediated metacognitive interpretation of conflict, presumably the upstream signals that lead to conflict can vary (and therefore lead to the differences identified here), while eliciting the same conscious experience of the mnemonic mismatch and its resolution. Thus, a number of possible sources of phenomenological discrepancy between the naturalistic déjà vu experience and its experimentally-generated counterpart exist, and remain to be explored. These discrepancies could perhaps be overcome by incorporating aspects of other successful déjà vu analogues with high stimulus face validity (e.g. Cleary et al., 2012; Cleary \& Claxton, 2018). The Cleary group's use of virtual navigation through immersive scenes make salient a 'physical' setting which participants frequently describe as a trigger of déjà vu (Brown, 2004), and thus has the potential to drive an experience associated with a perceptual environment that more closely resembles that in the naturalistic experience. The current DRM-based procedure does not lend itself so readily to using dynamic, interactive scenes as stimuli, but we hope that other future paradigms will pull together the most important components of the range of experimental analogues that exist today. In doing this, we believe we can all continue to improve the theoretical and face-validity of the déjà vu-like experiences we generate in the lab.

Whilst this experiment adds to a small body of evidence supporting the memory conflict account of déjà vu, the broader implications extend beyond experimental work and into naturalistic and clinical domains. It is a paradox that a putative memory error such as déjà vu should decline with age (for reviews, see Brown, 2003, 2004; Moulin, 2018). However, when it is considered that frontal deficits, both in terms of frontal function and brain health, are associated with ageing (e.g. Park, Polk, Mikels, Taylor, \& Marshuetz, 2001), this longitudinal pattern is consistent with a memory conflict account. A degraded cognitive system that is less capable of detecting minor inconsistencies in mnemonic information should experience fewer episodes of déjà vu, albeit without particularly noticeable consequences as long as the 
memory system is basically healthy. By extension though, déjà vécu, a dementia-related clinical manifestations of déjà vu, is characterised by behavioural change such as withdrawing from activities patients find erroneously familiar (Moulin et al., 2005). The associated memory failure, coupled with marked frontal dysfunction including confabulation, points to a frontal system where the deficit goes beyond conflict detection failure and supports and propagates highly unusual aberrations in memory signalling (e.g. faulty novelty signalling that is interpreted as recollection; O'Connor, Lever, et al., 2010). We know that frontal structures support a range memory functions, and the particular phenomenology of déjà vu and related disorders may be equally influenced by the health of these systems.

In summary, we provide the first neuroscientific evidence in healthy volunteers for the consideration of déjà vu as an experience driven by mnemonic conflict. Midline frontal regions involved in cognitive control, monitoring and conflict resolution may play a role in the phenomenology of the experience, though much remains to be elucidated about how the various components leading to mnemonic conflict are integrated into the conscious experience. Nonetheless, metacognitive evaluation of conflicting sources of mnemonic information appears important to this puzzling, compelling experience.

\section{Acknowledgements}

This research was funded by an anonymous donation to the School of Psychology and Neuroscience at the University of St Andrews. 


\section{References}

Adachi, N., Koutroumanidis, M., Elwes, R. D. C., Polkey, C. E., Binnie, C. D., Reynolds, E. H., . . Panayiotopoulos, C. P. (1999). Interictal 18FDG PET Findings in Temporal Lobe Epilepsy With Déjà vu. The Journal of Neuropsychiatry and Clinical Neurosciences, 11(3), 380-386. doi:10.1176/jnp.11.3.380

Ahrens, J., Geveci, B., Law, C., Hansen, C., \& Johnson, C. (2005). 36-ParaView: An EndUser Tool for Large-Data Visualization. The Visualization Handbook, 717.

Bartolomei, F., Barbeau, E. J., Nguyen, T., McGonigal, A., Régis, J., Chauvel, P., \& Wendling, F. (2012). Rhinal-hippocampal interactions during déjà vu. Clinical Neurophysiology, 123(3), 489-495. doi:10.1016/j.clinph.2011.08.012

Botvinick, M., Nystrom, L. E., Fissell, K., Carter, C. S., \& Cohen, J. D. (1999). Conflict monitoring versus selection-for-action in anterior cingulate cortex. Nature, 402(6758), 179-181. doi:10.1038/46035

Brainard, D. H. (1997). The Psychophysics Toolbox. Spat Vis, 10(4), 433-436. doi:10.1163/156856897X00357

Brázdil, M., Mareček, R., Urbánek, T., Kašpárek, T., Mikl, M., Rektor, I., \& Zeman, A. (2012). Unveiling the mystery of déjà vu: The structural anatomy of déjà vu. Cortex, 48(9), 1240-1243. doi:10.1016/j.cortex.2012.03.004

Brown, A. S. (2003). A review of the déjà vu experience. Psychological Bulletin, 129(3), 394-413. doi:10.1037/0033-2909.129.3.394

Brown, A. S. (2004). The déjà vu experience. Hove: Psychology Press.

Brown, A. S., \& Marsh, E. J. (2009). Creating Illusions of Past Encounter Through Brief Exposure. Psychological Science, 20(5), 534-538. doi:10.1111/j.14679280.2009.02337.x

Bunge, S. A., Hazeltine, E., Scanlon, M. D., Rosen, A. C., \& Gabrieli, J. D. E. (2002). Dissociable Contributions of Prefrontal and Parietal Cortices to Response Selection. NeuroImage, 17(3), 1562-1571. doi:10.1006/nimg.2002.1252 
Cabeza, R., Mazuz, Y. S., Stokes, J., Kragel, J. E., Woldorff, M. G., Ciaramelli, E., . . Moscovitch, M. (2011). Overlapping Parietal Activity in Memory and Perception: Evidence for the Attention to Memory Model. Journal of cognitive neuroscience, 23(11), 3209-3217. doi:10.1162/jocn_a_00065

Carter, C. S., \& van Veen, V. (2007). Anterior cingulate cortex and conflict detection: An update of theory and data. Cognitive, Affective, \& Behavioral Neuroscience, 7(4), 367-379. doi:10.3758/cabn.7.4.367

Cleary, A. M., Brown, A. S., Sawyer, B. D., Nomi, J. S., Ajoku, A. C., \& Ryals, A. J. (2012). Familiarity from the configuration of objects in 3-dimensional space and its relation to déjà vu: A virtual reality investigation. Consciousness and Cognition, 21(2), 969-975. doi:10.1016/j.concog.2011.12.010

Cleary, A. M., \& Claxton, A. B. (2018). Déjà Vu: An Illusion of Prediction. Psychological Science, 29(4), 635-644. doi:10.1177/0956797617743018

Cleary, A. M., \& Reyes, N. L. (2009). Scene recognition without identification. Acta Psychologica, 131(1), 53-62. doi:10.1016/j.actpsy.2009.02.006

Cleary, A. M., Ryals, A. J., \& Nomi, J. S. (2009). Can déjà vu result from similarity to a prior experience? Support for the similarity hypothesis of déjà vu. Psychonomic Bulletin and Review, 16(6), 1082-1088. doi:10.3758/PBR.16.6.1082

Cox, R. W. (1996). AFNI: Software for analysis and visualization of functional magnetic resonance neuroimages. Computers and Biomedical Research, 29, 162-173. doi:10.1006/cbmr.1996.0014

Cox, R. W., \& Hyde, J. S. (1997). Software tools for analysis and visualization of FMRI Data. NMR in Biomedicine, 10, 171-178. doi:10.1002/(SICI)10991492(199706/08)10:4/5\%3C171::AID-NBM453\%3E3.0.CO;2-L

Gold, S., Christian, B., Arndt, S., Zeien, G., Cizadlo, T., Johnson, D. L., . . . Andreasen, N. C. (1998). Functional MRI statistical software packages: a comparative analysis. Human Brain Mapping, 6, 73-84. doi:10.1002/(SICI)1097-0193(1998)6:2\%3C73::AIDHBM1\%3E3.0.CO;2-H 
Guedj, E., Aubert, S., McGonigal, A., Mundler, O., \& Bartolomei, F. (2010). Déjà-vu in temporal lobe epilepsy: Metabolic pattern of cortical involvement in patients with normal brain MRI. Neuropsychologia, 48(7), 2174-2181.

doi:10.1016/j.neuropsychologia.2010.04.009

Jaeger, A., Konkel, A., \& Dobbins, I. G. (2013). Unexpected novelty and familiarity orienting responses in lateral parietal cortex during recognition judgment. Neuropsychologia, 51(6), 1061-1076. doi:10.1016/j.neuropsychologia.2013.02.018

Jersakova, R., Moulin, C. J. A., \& O’Connor, A. R. (2016). Investigating the Role of Assessment Method on Reports of Déjà Vu and Tip-of-the-Tongue States during Standard Recognition Tests. PLOS ONE, 11(4), e0154334. doi:10.1371/journal.pone.0154334

Konishi, S., Wheeler, M. E., Donaldson, D. I., \& Buckner, R. L. (2000). Neural Correlates of Episodic Retrieval Success. NeuroImage, 12(3), 276-286. doi:10.1006/nimg.2000.0614

Madan, C. (2015). Creating 3D visualizations of MRI data: A brief guide. F1000Research, 4, 466. doi:10.12688/f1000research.6838.1

Mandler, G. (1980). Recognizing: The judgment of previous occurrence. Psychological Review, 87(3), 252-271. doi:10.1037/0033-295X.87.3.252

Martin, C. B., Mirsattari, S. M., Pruessner, J. C., Pietrantonio, S., Burneo, J. G., HaymanAbello, B., \& Köhler, S. (2012). Déjà vu in unilateral temporal-lobe epilepsy is associated with selective familiarity impairments on experimental tasks of recognition memory. Neuropsychologia, 50(13), 2981-2991. doi:10.1016/j.neuropsychologia.2012.07.030

McDermott, K. B., Gilmore, A. W., Nelson, S. M., Watson, J. M., \& Ojemann, J. G. (2017). The parietal memory network activates similarly for true and associative false recognition elicited via the DRM procedure. Cortex, 87, 96-107. doi:10.1016/j.cortex.2016.09.008 
Metcalfe, J., \& Schwartz, B. L. (2016). The ghost in the machine: Self-reflective consciousness and the neuroscience of metacognition. In J. Dunlosky \& S. K. Tauber (Eds.), The Oxford Handbook of Metacognition (pp. 407-424). Oxford: Oxford University Press.

Mill, R. D., Cavin, I., \& O'Connor, A. R. (2015). Differentiating the Functional Contributions of Resting Connectivity Networks to Memory Decision-making: fMRI Support for Multistage Control Processes. Journal of cognitive neuroscience, 27(8), 1617-1632. doi:10.1162/jocn_a_00808

Moulin, C. J. A. (2018). The Cognitive Neuropsychology of Déjà vu. Oxford: Routledge.

Moulin, C. J. A., Conway, M. A., Thompson, R. G., James, N., \& Jones, R. W. (2005). Disordered memory awareness: recollective confabulation in two cases of persistent déjà vecu. Neuropsychologia, 43(9), 1362-1378. doi:10.1016/j.neuropsychologia.2004.12.008

O'Connor, A. R., Barnier, A. J., \& Cox, R. E. (2008). Deja vu in the laboratory: a behavioral and experiential comparison of posthypnotic amnesia and posthypnotic familiarity. Int J Clin Exp Hypn, 56(4), 425-450. doi:10.1080/00207140802255450

O'Connor, A. R., Han, S., \& Dobbins, I. G. (2010). The Inferior Parietal Lobule and Recognition Memory: Expectancy Violation or Successful Retrieval? The Journal of Neuroscience, 30(8), 2924-2934. doi:10.1523/jneurosci.4225-09.2010

O'Connor, A. R., Lever, C., \& Moulin, C. J. A. (2010). Novel insights into false recollection: A model of déjà vécu. Cognitive Neuropsychiatry, 15(1-3), 118-144. doi:10.1080/13546800903113071

O’Connor, A. R., \& Moulin, C. J. A. (2010). Recognition Without Identification, Erroneous Familiarity, and Déjà Vu. Current Psychiatry Reports, 12(3), 165-173. doi:10.1007/s11920-010-0119-5

Park, D. C., Polk, T. A., Mikels, J. A., Taylor, S. F., \& Marshuetz, C. (2001). Cerebral aging: integration of brain and behavioral models of cognitive function. Dialogues in Clinical Neuroscience, 3(3), 151-165. 
Poldrack, R. A. (2011). Inferring mental states from neuroimaging data: From reverse inference to large-scale decoding. Neuron, 72(5), 692-697. doi:10.1016/j.neuron.2011.11.001

Ridderinkhof, K. R., Ullsperger, M., Crone, E. A., \& Nieuwenhuis, S. (2004). The Role of the Medial Frontal Cortex in Cognitive Control. Science, 306(5695), 443-447. doi:10.1126/science.1100301

Roediger, H. L., \& McDermott, K. B. (1995). Creating false memories: Remembering words not presented in lists. Journal of Experimental Psychology: Learning, Memory, and Cognition, 21(4), 803-814. doi:10.1037/0278-7393.21.4.803

Schumacher, E. H., Elston, P. A., \& D'Esposito, M. (2003). Neural Evidence for Representation-Specific Response Selection. Journal of cognitive neuroscience, 15(8), 1111-1121. doi:10.1162/089892903322598085

Urquhart, J. A., \& O'Connor, A. R. (2014). The awareness of novelty for strangely familiar words: a laboratory analogue of the déjà vu experience. PeerJ, 2, e666. doi:10.7717/peerj.666

Vignal, J.-P., Maillard, L., McGonigal, A., \& Chauvel, P. (2007). The dreamy state: hallucinations of autobiographic memory evoked by temporal lobe stimulations and seizures. Brain, 130(1), 88-99. doi:10.1093/brain/aw1329

Weinand, M. E., Hermann, B., Wyler, A. R., Carter, L. P., Oommen, K. J., Labiner, D., . . Herring, A. (1994). Long-Term Subdural Strip Electrocorticographic Monitoring of Ictal Déjà Vu. Epilepsia, 35(5), 1054-1059. doi:10.1111/j.1528-1157.1994.tb02554.x

Yushkevich, P. A., Piven, J., Hazlett, H. C., Smith, R. G., Ho, S., Gee, J. C., \& Gerig, G. (2006). User-guided 3D active contour segmentation of anatomical structures: significantly improved efficiency and reliability. NeuroImage, 31(3), 1116-1128. doi:10.1016/j.neuroimage.2006.01.015 